\section{Role of procalcitonine, hsCRP and 16SrRNA in diagnosis of culture negative endocarditis}

\author{
Asmaa O. Ahmed ${ }^{1}$, Sohair M. Ahmed ${ }^{1}$, Ayman K. M. \\ Hassan ${ }^{2}$, Mohammed E. Ali ${ }^{3}$ and Safaa A. Samir ${ }^{1}$
}

\author{
${ }^{1}$ Department of Clinical Pathology, Faculty of Medicine, Assiut \\ University, Assiut, Egypt. \\ ${ }^{2}$ Department of Cardiology, Faculty of Medicine, Assiut \\ University, Assiut, Egypt. \\ ${ }^{3}$ Department of Microbiology and Immunology, Faculty of \\ pharmacy, Al-Azhar University, Assiut, Egypt.
}

The Egyptian Journal of Immunology Volume 28 (4), 2021: 233-240. www.Ejimmunology.org
Corresponding author: Safaa A. Samir, Department of Clinical Pathology, Faculty of Medicine, Assiut University, Assiut, Egypt.

Email: safaa.samir191@gmail.com.

\begin{abstract}
Infective endocarditis infects the heart's inner surface, mostly the valves. It is a lethal disease with a high rate of morbidity and fatality. It mostly caused by bacteria, but fungi can also cause it. Microbiological diagnosis relies on blood culture. Molecular and biochemical indicators add to diagnosis, particularly in culture-negative patients. This study aimed to decide the role of $16 \mathrm{~s}$ rRNA, procalcitonin, and high sensitivity $C$ reactive protein (hSCRP) in the diagnosis of culture negative infective endocarditis (CNIE) in Assiut university hospitals. This cross-sectional study included 60 patients who were admitted to cardiac hospitals with suspected infective endocarditis according to modified DUKE criteria. A group of 20 apparently healthy subjects served as a control group for investigation of inflammatory biomarkers. We performed blood culture, biochemical markers and molecular investigations. Of the 60 patients, there were 46 (76.7\%) culture positives and 14 (23.3\%) culture negatives. Staphylococcus aureus was the most prevalent pathogen in culture positive patients, followed by enterococcus and klebsiella. Linezolid and imipenem were the most sensitive antibiotics for Gram-positive and Gram-negative bacteria, respectively. Amplification of bacterial $16 \mathrm{~S}$ rRNA gene, after DNA extraction from whole blood samples, was positive in $11 / 14$ cases $(78.5 \%)$ of culture negative patients and in $42 / 46$ cases $(91.3 \%)$ of culture positive patients. The range of procalcitonin in culture positive patients was $(0.32-89) \mathrm{ng} / \mathrm{ml}$, significantly higher than in culture negative patients $(0.02-8.8) \mathrm{ng} / \mathrm{ml}$, and in the control group $(0.01-0.08) \mathrm{ng} / \mathrm{ml}$. hsCRP showed the same pattern. In conclusion, our data suggest that PCR was the most accurate diagnostic tool for diagnosing CNIE, followed by procalcitonin, and hSCRP, respectively.
\end{abstract}

Keywords: endocarditis; blood culture; culture negative; 16s rRNA.

Date received: 08 July 2021; accepted: 27 September 2021.

\section{Introduction}

Infective endocarditis (IE) is a potentially lethal infection of the inner surface of the heart, mainly the valves. ${ }^{1}$ Despite the possibility of fungal endocarditis, it is almost always caused by bacteria. ${ }^{2}$ While anyone can get IE, there are specific risk factors that are well known. 
Patients with valvar defects, artificial valves, cardiac implantable electric devices (CIEDs), and intravenous drug users are among the most important risk factors. ${ }^{3}$ The clinical picture might be disparate depending on the host as well as the causative germ. The diagnosis is often difficult, requiring a mix of clinical, microbiological, and echocardiographic evidence. ${ }^{4}$ Over the previous five decades, there have been many changes in the clinical spectrum, etiological organism profile, and diagnostic procedures, as well as major geographic variations in risk factors. ${ }^{5}$ In affluent countries, more than half of those who have IE episodes have normal heart valves, but rheumatic heart disease (RHD) accounts for only $10 \%$ of IE cases. However, RHD is still the most common predisposing condition in developing countries. ${ }^{6}$ Blood culture is still the gold standard for diagnosing IE and determining which antibiotics to use. Culture-positive or culture-negative infective endocarditis are two different classifications. Prior antibiotic administration is by far the most common cause of "culture-negative" endocarditis. Some microorganisms might take longer time to grow on culture media, and these organisms are known as fastidious because they have stringent growth needs. Pathogens such as Aspergillus niger, Brucella species, Coxiella burnetii, Chlamydia species, and HACEK bacteria (Haemophilus niger, Aggregatibacter actinomycetemcomitans, Cardiobacterium hominis, Eikenella corrodens, and Kingella kingae) are just a few examples. ${ }^{7}$ Patients may be incorrectly labelled as "culture-negative" endocarditis (CNE) due to a delay in growth and detection of these organisms. NE's next steps are as follows: Incubation for a longer time, special media, serological techniques, molecular diagnostic approaches, inflammatory biomarkers such as procalcitonin (PCT) and high sensitivity C-reactive protein (hsCRP). ${ }^{8}$ The changing demography of the disease in terms of patient profile and microbiology is one of the key hurdles in treating IE. Consequently, the aim of this study was to detect the role of 16s rRNA, procalcitonin, and hSCRP in the diagnosis of culture negative infective endocarditis (CNIE) in Assiut university hospitals.

\section{Patients and Methods}

This cross-sectional study was conducted in the Microbiological Unit of the Clinical Pathology Department of Assiut University Hospitals, during September 2019 to September 2020. A total of 60 patients suspected of having infective endocarditis were admitted to cardiac hospitals. Their ages ranged from 1.5 to 64 years old. The study protocol was reviewed and approved by the ethical committee of the Faculty of Medicine, Assiut University (IRB 17100460; 3/2018). The study goals were explained to all the subjects and to guardian of infants and children, and they gave their consent orally. In addition, for school age children (6-18 years of age), the blood collection method and study goals were explained in simple language, and willing to provide blood sample was taken as child assent.

All patients were subjected to the following:

\section{Microbiological testing}

A total of 5-10 mL of blood was obtained for adults and 2-3 $\mathrm{mL}$ for children for blood culture under aseptic conditions ${ }^{9}$ and put in blood culture bottle of BacT/ALERT (bioMérieux diagnostics, Lyon, France) which is an automated microbial detection system ${ }^{10}$. To avoid contamination, we withdrawn two samples from two different veins at a time. At interval of at least one hour, we repeated blood sample collection three times. All bottles were incubated in BacT/ALERT for 21 days for negative samples. The positive blood cultures on BacT/ALERT were subjected to identification of the organism by two methods: 1) a manual method, by culture on one plate of blood agar, chocolate agar, MacConkeys agar, Mannitol agar, EMB agar, and bilesculin agar; and on 2 plates of Sabaroud's agar, one was incubated at $25^{\circ} \mathrm{C}$ (room temperature) and the other at $37^{\circ} \mathrm{C}$, according to the lab's SOP (standard operating procedure of the USA Environmental Protection Agency) for identification of bacterial type based on morphology, and CLSI 2019 (the Clinical Standard Institute) for antibiotic susceptibility. 2) an automated method using the VITEK 2 COMPACT15 system (BioMerieux diagnostics, Lyon, France), according to the 
manufacturer's instructions, to confirm the bacterial identification and antibiotic susceptibility of the identified organism. ${ }^{11}$

\section{Biomarker investigations}

A group of 20 apparently healthy persons, age and sex cross matched with patients, formed a control group. Three $\mathrm{ml}$ blood were collected from each patient and control subject into plain tube without anticoagulant for assessing procalcitonin, using an immunoassay analyzer (cobas E 411, Roche Diagnostics) ${ }^{12}$ and hsCRP using an integrated system (Dimention xpand 2004083091, Siemens Healthineers Diagnostics, USA). ${ }^{13}$ Blood was allowed to clot for 15 minutes at $37{ }^{\circ} \mathrm{C}$ and serum was separated by centrifugation at $1560 \mathrm{Xg}$ for 10 minutes then kept at $-20^{\circ} \mathrm{C}$ until used.

\section{Molecular investigations}

All patient's blood samples were subjected to broad range bacterial 16s rRNA PCR assay as the following: a) DNA extraction: Bacterial DNA was extracted from EDTA blood using (QIAamp DNA Blood Mini kit) (QIAGEN, Hilden, Germany) as described by the manufacturer. For testing the yield and quality of the extracted DNA, DNA samples were electrophoresed on $2 \%$ agarose gel. ${ }^{14}$ For PCR amplification of 16s rRNA genes, five microliters of the extracted DNA were amplified in a $25 \mu$ volume PCR reaction using an oligonucleotide primer set (designed according the USA National Center for Biotechnology Information; NCBI). The forward primer was: CGGTCCAGACTCCTACGGGAGGCA GCA; and reverse primer: GCGTGGACTACCA GGGTATCTAATCC. The PCR reaction consisted of $12.5 \mu$ l2x Tag PCR Master Mix, one $\mu$ l of each forward and reverse primers, and PCR grade water to a final volume of $25 \mu \mathrm{l}$. The PCR amplification consisted of an initial denaturation step at $95 \mathrm{C}$ for $5 \mathrm{~min}$ followed by 35 cycles of denaturation at $95 \mathrm{C}$ for $30 \mathrm{~s}$, annealing at $65 \mathrm{C}$ for $30 \mathrm{~s}$, extension at $72 \mathrm{C}$ for $30 \mathrm{~s}$ and final extension at $72 \mathrm{C}$ for $10 \mathrm{~min}^{15}$. The PCR products were electrophoresed through agarose gel $2 \%$. Molecular sizes of the amplicons were determined by comparison to a 100 bp DNA ladder. A positive result was inferred by detection of 480 bp band ${ }^{16}$.

\section{Statistical analysis}

Statistical package for social science (SPSS) version 11 was used for data base construction, management and analysis. Quantitative data were summarized in the form of mean \pm SD. Qualitative data were summarized using frequencies and percentages. Student's t-test was done for comparison between two means and one-way ANOVA for more than two means. Probability ( $p$ value) less than 0.05 was considered significant. Accuracy of hsCRP, PCT and PCR in diagnosis of culture negative infective endocarditis was assessed by using receiver operator characteristics (ROC) curve.

\section{Results}

The study included 60 patients with suspected infective endocarditis based on the modified Duke criteria.

\section{Clinical and demographic data of studied group}

The mean age of enrolled patients was $30.5 \pm 16.2$ years with a range of (1.5-64 years). Of the 60 studied patients, 33 (55\%) patients were males and 27 (45\%) females. The most frequent risk factors for infective endocarditis were rheumatic heart disease in 20 patient (33.3\%), diabetes mellitus in 19 patients (31.6\%), 18 (30\%) with prosthetic valve, $11(18.3 \%)$ on hemodialysis and $8(13.3 \%)$ with congenital heart defect (Figure 1). 


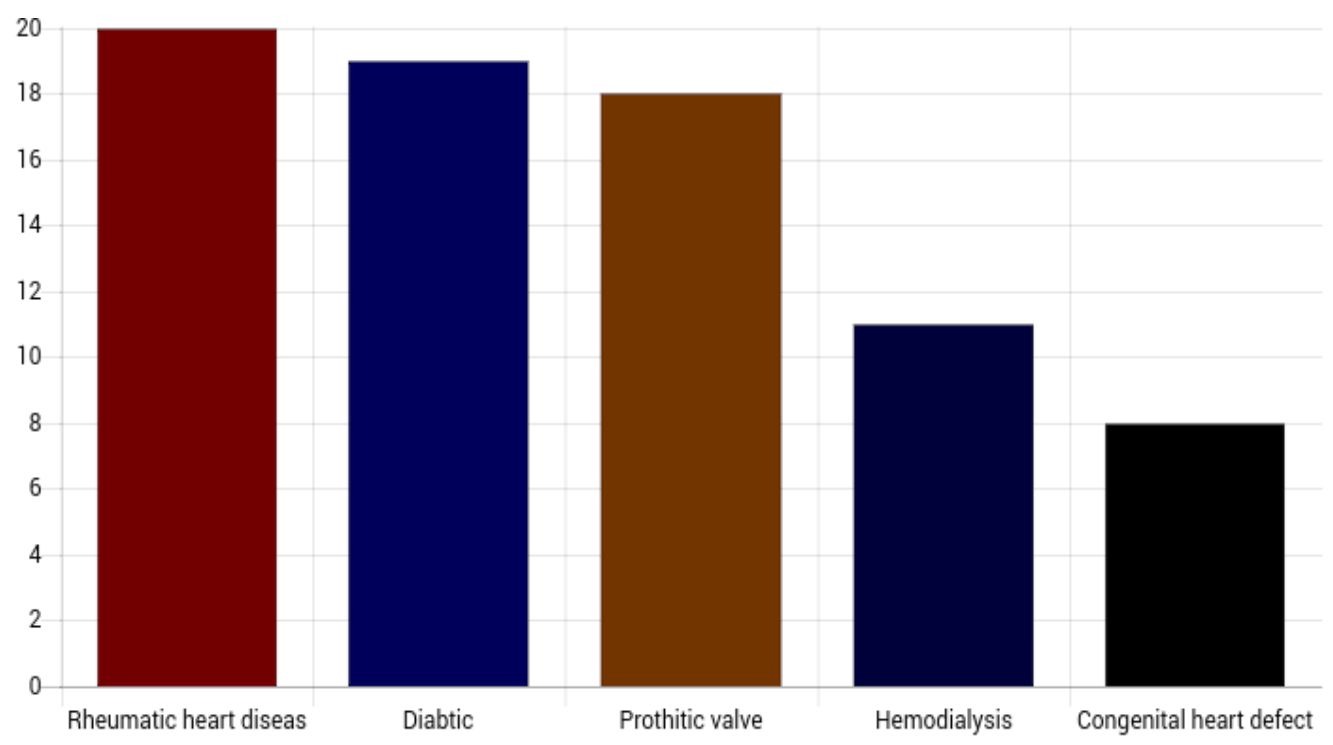

Figure 1. Underlying predisposing factors in study patients

\section{Microbiological results}

Positive blood culture samples were identified by conventional culture method according to SOPs of the lab and Vitek2 compact system. The results of organism type obtained by both methods were identical. Of the 60 patients, blood cultures of samples from 46 (76.7\%) were positive and negative in $14(23.3 \%)$ samples. Of the 46 culture positive patients, 10 patients had mixed organisms. So, the total number of detected organisms were 59 organisms; 46
(78\%) were gram positive organism while only 13 (22\%) were gram negative organism.

The result of organism differentiation of culture positive patients

There were 46 (78\%) of gram-positive organism and $13(22 \%)$ of gram-negative organism. The most common microorganism causing infective endocarditis was Staphylococcus aureus 28 (47.4\%) followed by Enterococcus 15 (25.4\%) then Klebsiella 8 (13.5\%) and their differentiation is shown in Table 1.

Table 1. Differentiation of organism in culture positive patients

\begin{tabular}{lc}
\hline Type of organism $(n=59)$ & Number of patients \\
\hline Gram positive organism & $46(78 \%)$ \\
-Staphylococcus aureus & $28(47.4 \%)$ \\
-Enterococcus & $15(25.4 \%)$ \\
-Staphylococcus hominis & $2(3.3 \%)$ \\
-Streptococcus & $1(1.6 \%)$ \\
Gram negative organism & $13(22 \%)$ \\
-Klebsiella & $8(13.5 \%)$ \\
-Acinetobacter & $5(8.4 \%)$ \\
\hline
\end{tabular}

\section{Biomarker investigations}

The results of biomarkers test of positive culture patients, negative culture patients and the control group are shown in Table 2. It was observed that the range and mean value of PCT and hsCRP in culture negative patients are less than those in culture positive patients, but more than the control group results. 
Table 2. Biomarker data in different study groups.

\begin{tabular}{lcccc} 
Biomarker & $\begin{array}{c}\text { Culture positive } \\
\text { patients }(n=46)\end{array}$ & $\begin{array}{c}\text { Culture negative } \\
\text { patients }(n=14)\end{array}$ & $\begin{array}{c}\text { Control group } \\
(n=20)\end{array}$ & $P$ value \\
\hline hsCRP $(\mathrm{mg} / \mathrm{dl})$ & $12.8 \pm 4.1$ & & & \\
Mean \pm SD & $(7.4-20)$ & $6.9 \pm 5.2$ & $0.3 \pm 0.1$ & NS \\
Range & & $(0.3-18)$ & $(0.1-0.7)$ & \\
\hline Procalcitonin $(\mathrm{ng} / \mathrm{ml})$ & $33.1 \pm 39.6$ & & & \\
Mean \pm SD & $(0.32-89)$ & $1.24 \pm 2.3$ & $0.04 \pm 0.02$ & $0.0001^{*}$ \\
Range & $(0.02-8.8)$ & $(0.01-0.08)$ & \\
\hline
\end{tabular}

*For comparison between the three groups. $P>0.05$ is no significant (NS).

\section{Molecular investigations}

Broad range PCR for amplification of bacterial 16S rRNA, of DNA extracted from whole blood samples of culture negative patients were positive in $11 / 14$ cases (78.5\%),(Figure 3 ) and $42 / 46$ cases $(91.3 \%)$ in culture positive patients (Figure 4).

Diagnostic accuracy of hsCRP, PCT and PCR in diagnosis of culture negative infective endocarditis

At cutoff $>10.2 \mathrm{mg} / \mathrm{dl}$, serum hsCRP had $45 \%$ sensitivity and $83.33 \%$ specificity for diagnosis of culture negative infective endocarditis with overall accuracy of $48.8 \%$. While PCT at cutoff $>$ $1.34 \mathrm{ng} / \mathrm{dl}$ had $57 \%$ sensitivity and $83.33 \%$ specificity for diagnosis of culture negative infective endocarditis with overall accuracy of 59.6\%. PCR had the best diagnostic accuracy (95.5\%) for diagnosis of culture negative infective endocarditis. It also, had 95\% sensitivity and $100 \%$ specificity. So, based on the ROC curve analysis, the most accurate method for diagnosis of CNIE was PCR with area under the curve of 0.97 (excellent accuracy) followed by PCT with area under the curve of 0.78 (Good accuracy) and then hsCRP with area under the curve of 0.72 (fair accuracy) Figure (5).

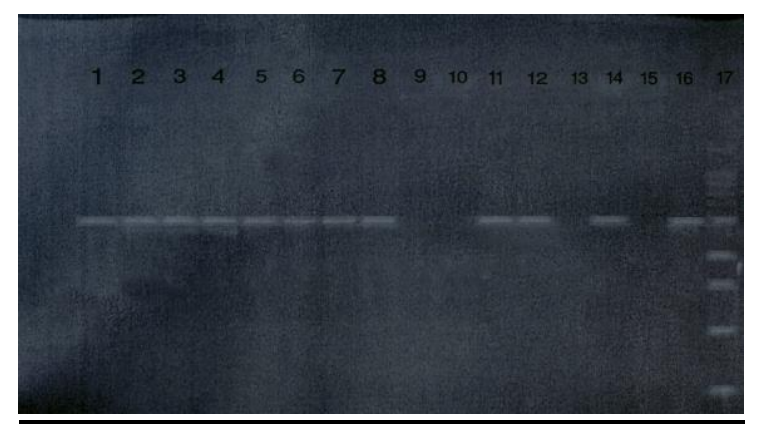

Figure 3. Agarose gel electrophoresis of PCR products of culture negative patients as resulted from universal amplification of 16S rRNA gene. Lane 17:100 bp DNA molecular weight ladder; Lane 16: positive control, Lane15: negative control, Lanes 14,12,11,8,7,6,5,4,3,2,1: positive PCR result of culture negative patients, Lanes 9,10,13: negative PCR result of culture negative patients.

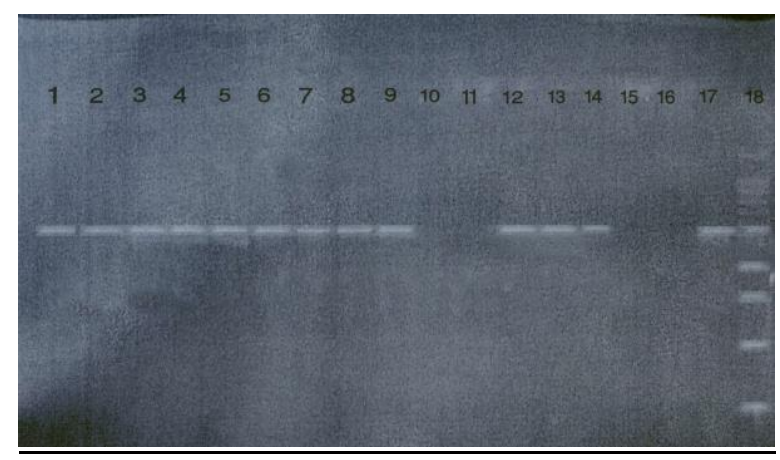

Figure 4. Agarose gel electrophoresis of PCR products of culture positive patients as resulted from universal amplification of $16 \mathrm{~S}$ rRNA gene. Lane 18:100 bp DNA molecular weight ladder, Lanes $1,2,3,4,5,6,7,8,9,12,13,14,17$ : positive PCR result of culture positive patients; Lanes 10,11,15,16: negative PCR result of culture positive patients. 


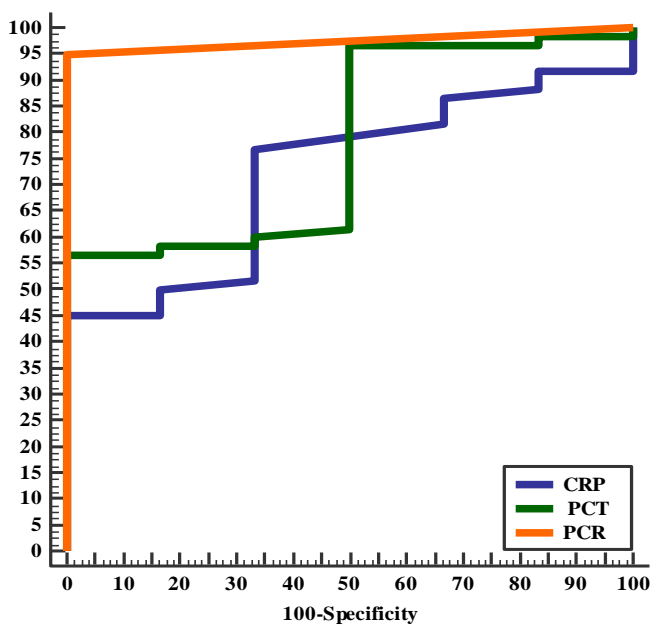

Figure 5. Roc curve analysis for PCT, hsCRP and PCR. Area under the curve: $>0.9=$ Excellent accuracy, $>0.8$ =Good accuracy, $>0.7=$ Fair accuracy, $\leq 0.7=$ Poor accuracy.

\section{Discussion}

The present study revealed that the most common risk factors associated with infective endocarditis patients were rheumatic heart disease (30.3\%), diabetes mellitus $(28,7 \%)$, prosthetic valve $(27,2 \%)$, hemodialysis $(18,1 \%)$, and CHD (12,1\%). These findings agreed with previous studies, reported history of rheumatic fever $(30 \%)$, prosthetic valve $(24 \%)$, and CHD $(8 \%)$ as common risk factors. ${ }^{17-18}$ However, another study revealed that chronic hemodialysis was the most common risk factor (26.7\%) for IE cases ${ }^{3}$. Also, another study found that diabetes mellitus was the most common risk factor (18.2\%) for IE. ${ }^{19}$

In this study the culture positive IE patients were $46(76.6 \%)$ and culture negative IE patients 14 (23.4\%). In contrast, a previous study revealed that culture positive IE patients accounted for $30.3 \%$ and culture negative for $69.7 \%{ }^{20}$

Changes in the epidemiology of IE have happened over the past several decades. ${ }^{21}$ In this study the three most common pathogens in culture positive infective endocarditis patients were: Staphylococcus aureus followed by Enterococcus then Klebsiella. In this regard, other studies reported that Staphylococcus aureus was the most common organism in culture positive IE patients. ${ }^{22,23}$

In this study the result of PCR revealed that for culture negative patients $16 \mathrm{~S}$ rRNA genes were amplified in $78.5 \%$ cases and in $91.3 \%$ for culture positive patients. So, in our study, there were 4 samples positive by blood culture but negative for amplification of 16S rRNA genes by $P C R$, and all were bacteria of gram-positive type. PCR-negative results in culture-positive patients especially with gram-positive pathogens were previously reported. ${ }^{24}$ The authors indicated that difficulties in breaking the cell wall of gram-positive organisms during sample preparation resulted in failure in the DNA extraction process. ${ }^{24,25,26}$

In our study PCR showed 95\% sensitivity, $100 \%$ specificity and $95.5 \%$ accuracy while a previous study showed $88.3 \%$ sensitivity, 92\% specificity and $91 \%$ accuracy. ${ }^{23}$ In a systematic review, it was concluded that PCT and hsCRP were neither sensitive nor specific for diagnosis of IE ${ }^{27}$. However, IE should be considered based on clinical information i.e., presence of fever, embolic phenomenon, new or changed heart murmur, risk factors for IE like valvar heart disease, intravenous drug abusers, and bacteremia with incomplete treatment especially with Staphylococcus. ${ }^{27}$

This study revealed that PCT showed a significant difference between IE patients and the control group, but CRP did not reach such significance. A previous study found that PCT had a critical role in the detection of potential IE infection in individuals with a history of cardiac issues who were referred with acute symptoms such as dyspnea. PCT had a predictive value that was linked to clinical outcome and could be utilized as a therapy guide. It also showed a strong link to a positive blood culture, which was an item in the diagnosis of the IE. PCT had a strong correlation with positive blood culture regardless of the pathogen or infection site. ${ }^{28}$

The PCR approach, as employed in our study, is inexpensive and simple to carry out in a clinical microbiological laboratory. Its costeffectiveness is measured not only in terms of material costs, but also in terms of the added value of early detection of bacteremia in patients and, as a result, the delivery of proper treatment, which lowers morbidity and death rates. However, DNA amplification approaches do not determine antibacterial susceptibility, which must be tested using traditional methods. 
Based on our study findings, we conclude that molecular approaches and biomarkers such as procalcitonin would add value in diagnosis of cases with infective endocarditis, particularly culture negative endocarditis. Nonetheless, PCR-based techniques cannot replace traditional culture procedures. The use of an automated blood culture device, as well as adequate blood culture collection under sterile conditions, would still aid in the identification of infective endocarditis.

\section{Author Contributions}

AOA; was responsible for methodology, analysis and interpretation of data, SMA; was responsible for project administration conception and design of the study and overall supervision, AKM; was responsible for review and editing of the study in addition to clinical evaluation of patients, MEA; was responsible for molecular method and experiments in addition to analysis and interpretation of data, SAS; was responsible for writing the original draft in addition to practical work.

\section{Declaration of Conflicting Interests}

The author(s) declared no potential conflicts of interest with respect to the research, authorship, and/or publication of this article.

\section{Funding}

The author(s) denies receipt of any financial support for the research, authorship, and/or publication of this article.

\section{Ethical approval}

The study protocol was reviewed and approved by the ethical committee of the Faculty of Medicine, Assiut University (IRB 17100460; 3/2018).

\section{Informed consent}

The study goals were explained to all the subjects and to guardian of infants and children, and they gave their consent orally. In addition, for school age children (6-18 years of age), the blood collection method and study goals were explained in simple language, and willing to provide blood sample was taken as child assent.

\section{References}

1. Merck, (2017). Infective Endocarditis Cardiovascular Disorders". Merck Manuals Professional Edition. September 2017.

2. Raja, K., Antony, M., and Ganapathi, (2018). Fungal infective endocarditis, J. The Academy of Microbiologists p. 96.

3. Mostaghim, Anahita, S, Hoi,Y., et al (2017). A retrospective epidemiologic study to define risk factors, microbiology, and clinical outcomes of infective endocarditis in a large tertiary-care teaching hospital. SAGE Open Medicine5: p. 2050312. DOL: 117741772.

4. Chirillo and Fabio (2021) New approach to managing infective endocarditis. 2020 Trends in Cardiovascular Medicine, 31(5):287-289.

5. Ren, Zuning,Mo., et al. (2019). A changing profile of infective endocarditis at a tertiary hospital in China: a retrospective study from 2001 to 2018. 2019. BMC Infectious Diseases, 19(1): 1-10.

6. Kundavaram, (2017). Trends of Infective Endocarditis in India: A South Indian Experience $J$ Cardiovasc Disease Res; 8(2):56-60. 2.

7. Del Giudice, C., Vaia, E., Liccardo, D., et al. (2021). Infective Endocarditis: A Focus on Oral Microbiota, Microorganisms p. 1218.

8. Kolb, M., Lazarevic, V., Emonet, S., et al. (2019). Next-generation sequencing for the diagnosis of challenging culture-negative endocarditis. Frontiers of Medicine 6: p. 203.

9. Linscott.A.J, Amyl.Leber editor, section 2, clinical microbiological procedure hand book. 2016.

10. Thorpe, T.C., Wilson, M., Turner, J., et al. (1990). BacT/Alert: an automated colorimetric microbial detection system.Journal of Clinical Microbiology. 28(7): 1608-1612.

11. Crowley, E., Bird, P., Fisher, K., et al. (2012). Evaluation of the VITEK 2 gram positive (GP) microbial identification test card: collaborative study. J Aoac International. 95(5): 1425-1432.

12. Becker, K., Nylen, E., White, J., et al. (2004). Procalcitonin and the calcitonin gene family of peptides in inflammation, infection, and sepsis: a journey from calcitonin back to its precursors. J Clinical Endocrinology \& Metabolism 89(4): 15121525.

13. VanLeeuwen, M.A., Van Rijswijk and Martin H J, (1994). Acute phase proteins in the monitoring of inflammatory disorders. Baillière's Clinical Rheumatology 8(3): 531-552.

14. Lahiri, D.K. and Schnabel, B. (1993). DNA isolation by a rapid method from human blood samples: effects of $\mathrm{MgCl} 2$, EDTA, storage time, and 
temperature on DNA yield and quality. Biochemical Genetics, VoL 3i, Nos. 7/8, 1(7-8): 321-328.

15. Srinivasan, R., Karaoz, U., Volegova, M., et al. (2015). Use of 16S rRNA gene for identification of a broad range of clinically relevant bacterial pathogens. PLOS ONE I DOI:10.1371/journal.pone.0117617

16. Ye J, Coulouris G, Zaretskaya I., et al. (2012). "Primer-BLAST: a tool to design target-specific primers for polymerase chain reaction." BMC Bioinformatics 13:134.

17. Thornhill, M.H., Jones, S., Prendergast, B., et al. (2018). Quantifying infective endocarditis risk in patients with predisposing cardiac conditions. European Heart Journal 39(7): p. 586-595.

18. Andersen, M., Holle, S, Klein, C., et al. (2020). Risk for infective endocarditis in bacteremia with Gram positive cocci Springer-Verlag GmbH Germany, 48(6): 905-912.

19. Kim, J, Lee, H, Ku, N., et al. (2020). Infective endocarditis at a tertiary care hospital in South Korea. Heart 107(2): 135-141.

20. Rizk, H, Elamragy, A, Youssef, G., et al. (2019). Clinical features and outcomes of infective endocarditis in Egypt: an 11-year experience at a tertiary care facility. The Egypt. Heart J 71(1): 17.

21. Vogkou, C, Vlachogiannis, N, Palaiodimos, L., et al. (2016). The causative agents in infective endocarditis: a systematic review comprising 33,214 cases. Eur J Clin Microbiol Infect Dis 35(8): 12271245.
22. Pant, S., Patel, N, Deshmukh, A., et al. (2015). Trends in infective endocarditis incidence, microbiology, and valve replacement in the United States from 2000 to 2011. J The Americ College of Cardiol. 65(19): 2070-2076.

23. Damlin, A., Westling, K., Maret, E., et al. (2019). Association between echocardiographic manifestations and bacterial infections in patients with infective endocarditis: a cohort study. BMC Infectious Diseases.

24. El-Kholy, A, El-Rachidi, N, El-Enany, M., et al. (2015). Impact of serology and molecular methods on improving the microbiologic diagnosis of infective endocarditis in Egypt. Springer-Verlag Berlin Heidelberg 43(5): 523-529.

25. Rovery C, Greub G, Lepidi H, et al. (2005). PCR detection of bacteria on cardiac valves of patients with treated bacterial endocarditis. J Clin Microbiol.; 43:163-7.

26. Rothman R, Majmudar M, Kelen G., et al. (2002). Detection of bacteremia in emergency department patients at risk for infective endocarditis using universal 16S rRNA primers in a decontaminated polymerase chain reaction assay. J Infect Dis; 186:1677-81.

27. Singh, M. and Koyfman, A. (2014). What is the role of procalcitonin in early diagnosis of infective endocarditis. Annals of Emergency Medicine 66(1): 25-26.

28. Schuetz, P., Daniels, L, Kulkarni, P., et al. (2016). Procalcitonin a new biomarker for the cardiologist. Inte J. cardiol. 223:390-397. 